\title{
Hepatocyte nuclear factor 4 alpha P2 promoter variants associate with insulin resistance
}

\author{
Riyadh Saif-Ali ${ }^{1}$, Roslan Harun ${ }^{1,2}$, S. Al-Jassabi³ and Wan Zurinah Wan Ngah ${ }^{1,2 \bowtie}$ \\ 1UKM Medical Molecular Biology Institute (UMBI); ${ }^{2}$ UKM Medical Center, University Kebangsaan, Malaysia; ${ }^{3}$ nstitute of Biological Sciences, Uni- \\ versity of Malaya, Malaysia
}

\begin{abstract}
This study aimed to investigate the associations of hepatocyte nuclear factor 4 (HNF4) alpha single nucleotide polymorphisms (SNPs) and haplotype with insulin resistance and metabolic syndrome parameters. Nine SNPs spanning the HNF4 alpha P2 promoter (rs4810424, rs1884613 and rs1884614) and coding region (rs2144908, rs6031551, rs6031552, rs1885088, rs1028583 and rs3818247) were genotyped in 160 subjects without diabetes or metabolic syndrome. The HNF4 alpha P2 promoter SNPs rs4810424, rs1884613 and rs1884614 were associated with insulin resistance $(p=0.017 ; 0.037$; $0.024)$ and body mass index (BMI) $(p=0.03 ; 0.035 ; 0.039)$. The intron 1D SNP rs2144908 was associated with highdensity lipoprotein cholesterol (HDLc) $(p=0.020)$ and the intron 9 SNP rs3818247 showed association with systolic $(p=0.02)$ and diastolic $(p=0.034)$ blood pressure. HNF4 alpha common haplotype CCCGTC associated with higher insulin resistance $(p=0.022)$, fasting blood glucose (FBG) $(p=0.035)$ and lower HDLc $(p=0.001)$. In conclusion, subjects with HNF4 alpha P2 variants and haplotypes have been shown to have a higher insulin resistance and are therefore at a higher risk for developing type 2 diabetes mellitus.
\end{abstract}

Keywords: insulin resistance, HNF4 alpha, single nucleotide polymorphisms, haplotypes

Received: 01 October, 2010; 28 March, 2011; accepted: 03 May, 2011; available on-line: 02 June, 2011

\section{INTRODUCTION}

Insulin resistance and beta cell dysfunction precede the development of intermediate hyperglycaemia and the subsequent type 2 diabetes mellitus (T2DM). Insulin resistance is a feature of a number of diseases including obesity, glucose intolerance, dyslipidemia and hypertension clustering in the so-called metabolic syndrome (Sesti, 2006) that together are an indicator of risk for type 2 diabetes mellitus. The prevalence of T2DM in school children is on the increase, which is associated with obesity, metabolic syndrome and insulin resistance $(\mathrm{Na}-$ than, 2007; Cali \& Caprio, 2008; Holst-Schumacher et al., 2009; Phillips \& Phillips, 2009; Amed et al., 2010). The peripheral insulin resistance refers to diminished insulinmediated uptake of glucose principally by skeletal muscle and adipose tissue, which depends mainly on the control of glucose transporter type 4 (GLUT4) expression and translocation to the plasma membrane (Leclercq et al., 2007). Hepatic insulin resistance refers to insufficient ability of insulin to suppress hepatic glucose production that account for hyperglycemia. Eighty percent of sub- jects converted to T2DM are insulin resistant (Haffner et al., 2000).

Hepatocyte nuclear factor 4 alpha (HNF4 alpha), mostly expressed in the liver and pancreatic beta cells, is a transcriptional factor essential for normal functioning of hepatocytes and endocrine pancreas (Parviz et al., 2003; Odom et al., 2004). HNF4 alpha controls the expression of several transcription factors (Gonzalez, 2008; Bolotin et al., 2010) and regulates several genes encoding components of insulin secretion and glucose metabolism (Stoffel \& Duncan 1997; Wang et al., 2000). Polymorphisms of HNF4 alpha gene may be associated with insulin resistance. The aim of this project was to study the association of HNF4 alpha P2 promoter SNPs and haplotypes with the insulin resistance and metabolic syndrome parameters (waist circumference, body mass index, fasting blood glucose, C-peptide, systolic and diastolic blood pressure, triglyceride, total cholesterol, LDL and HDL cholesterol).

\section{MATERIALS AND METHODS}

Ethical approval was obtained from the National University of Malaysia Research and Ethics committee. The subjects were randomly approached through distribution of brochures to office letterboxes around Cheras area. The Hospital and Faculty of Medicine staffs of University Kebangsaan Malaysia were approached. Brochures were also given to participants to distribute to their relatives and friends. In two years, around nine hundred subjects responded, but after several tries, only 262 were included. After biochemical analysis of their blood, 160 of the 262 were found without diabetes or metabolic syndrome

Biochemical analysis. Kits for the measurement of glucose, triglyceride, total cholesterol and HDL cholesterol (reference number 10260, 10724, 10028 and 10018, respectively) were purchased from the Human Company (Human GmbH, Wiesbaden, Germany). Human company Elevated control sera (Humatrol P Reference number 13512) was used as quality control for these parameters. C-peptide was measured in an automated quantitative immunoassay analyzer (Immulite, DPC, Los Angeles,

e-mail: zurina@medic.ukm.my

Abbreviations: BMI, body mass index; BP, blood pressure; DHPLC, denaturing high-performance liquid chromatography; BG, fasting blood glucose; HNF4 alpha, hepatocyte nuclear factor 4 alpha; SNP, single nucleotide polymorphism; HDLc, high-density lipoprotein cholesterol, F T2DM, type 2 diabetes mellitus; LDLc, low-density lipoprotein cholesterol; PCR, polymerase chain reaction; TG, triglyceride 
USA) using IMMULITE C-peptide kit (catalogue number LKPE1). Insulin resistance was calculated using the Homeostasis Model Assessment (HOMA2) Calculator v2.2. This program uses fasting C-peptide or insulin and blood glucose measurement to calculate insulin resistance.

Genotype analysis. HNF4 alpha SNPs rs4810424, rs1884613, rs1884614, rs2144908, rs6031551, rs6031552, rs1885088, rs1028583 and rs3818247 were selected for genotypic analysis in based on published results from the Finnish and Ashkenazi Jewish studies (Love-Gregory et al., 2004; Silander et al., 2004). All SNPs in this research were amplified by in an Icycler thermocycler (Bio-Rad Laboratories, Inc., Richmond, CA, USA) using a $96 \mathrm{mi}-$ crowell plate. Touchdown PCR was applied for all SNPs except rs1884613 which was amplified and identified by allele specific PCR (AS-PCR). Restriction enzymes (New England Biolabs, USA) were used for identifying the genotypes of rs4810424 (BstXI), rs1884614 (BsgI), rs6031551 (MseI), rs6031552 (CvikI-1), rs1885088 (Sau96) and rs1028583 (Pst $\mathrm{I})$. The complete procedures of PCR amplification and genotyping of the SNPs are available from the authors.

Rs2144908 and rs3818247 were genotyped by denaturing high-performance liquid chromatography (DHPLC) (Varian Inc, Palo Alto, CA, USA). A recommendation for running rs 2144908 and rs 3818247 at $60^{\circ} \mathrm{C}$ and $58^{\circ} \mathrm{C}$, respectively, was obtained when these SNPs PCR amplified sequences were uploaded to the DHPLC melt program (http://insertion.stanford.edu/melt.html). These recommended temperatures were confirmed experimentally by running $5 \mu$ l of PCR products of these SNPs on the DHPLC at two degrees below and above. Separated peaks of heteroduplex and homoduplex DNA from heterozygous samples of rs2144908 and rs3818247 were detected at $59^{\circ} \mathrm{C}$ and $57.5^{\circ} \mathrm{C}$, respectively, using DHPLC program gradient buffer and time. Known heterozygous, homozygous minor and homozygous major genotypes of rs2144908 and rs3818247 (these samples were sequenced) were included into the runs as quality controls which were run at $59^{\circ} \mathrm{C}$ and $57.5^{\circ} \mathrm{C}$, respectively. Some samples were sequenced by automated 3130xl Genetic Analyzer (Applied Biosystems, Foster City, CA, USA) using terminator cycle sequencing kit v3.1 to confirm the allele specific PCR and DHPLC results.

Statistics. HelixTree 6.0.1 SNP and Variation Suite for Genetic statistics was used to study the deviation of SNPs from the Hardy-Weinberg equilibrium and to study the linkage disequilibrium between SNPs and construct haplotypes and diplotypes of related SNPs. Other statistical analyses were done by SPSS 11.5 program. The missing data were listwise deleted (when any of the variables were missing, the entire observation was omitted from the analysis). The BMI, waist, systolic and diastolic blood pressure, fasting blood glucose, C-peptide, insulin resistance, total cholesterol, triacylglycerol, HDL and LDL cholesterol data were log transformed because they were not normally distributed. These parameters' means and $95 \%$ confidence intervals were transformed back and reported, and were called geometric means.

The linearity of correlation of metabolic syndrome parameters: insulin resistance, C-peptide, glucose, BMI, waist, systolic BP, diastolic BP, TG, total cholesterol, HDL cholesterol and LDL cholesterol (dependent variables) were analyzed by bivariate correlations, resulting in three sets of dependent variables. The first set was insulin resistance, C-peptide, fasting blood glucose, BMI, waist, triglyceride, and HDL cholesterol. The second set was total cholesterol and LDLc and the third set was systolic and diastolic blood pressure. The association of HNF4 alpha SNPs, haplotypes and diplotypes with these three sets of dependent variables was evaluated by multivariate analyses (general linear model) adjusted for age, sex and race as covariates. Wilks' Lambda test was used for more than two groups of independent variables. Hotelling's Trace was used for two groups of independent variable. The difference between means was considered significant if $p$-value $<0.05$.

\section{RESULTS}

The demographic and biochemical parameters of the subjects are summarized in Table 1 . HelixTree program showed no deviation of all the SNPs included in this study from Hardy-Weinberg equilibrium. Five SNPs showed association with metabolic syndrome parameters: rs4810424, rs1884613, rs1884614, rs2144908 and rs3818247, whereas rs6031551, rs6031552 and rs1028583 showed no association. The homozygous minor and heterozygous genotypes frequencies of rs1885088 were very low in our subjects ( 0.0 and 0.03 , respectively) thus the association of this SNP with metabolic syndrome parameters was not studied.

\section{Association of P2 promoter SNP rs4810424 with metabolic syndrome parameters}

Multivariate analyses of this SNP with the three sets of metabolic syndrome parameters showed a significant Wilks' Lambda test $(\mathrm{P}=0.039)$ with the first set (insulin resistance, C-peptide, FBG, TG, HDLc, waist and $\mathrm{BMI}$ ) whereas the second set (total and LDL cholesterol) and the third set (systolic and diastolic blood pressure) were non-significant (Table 2). The subjects having

Table 1. Demography and biochemical parameters of subjects

\begin{tabular}{llc}
\hline Parameters & & $\mathrm{n}=160$ \\
\hline Gender & Male \% & 40.6 \\
& Female \% & 59.4 \\
races & Malay \% & 63.7 \\
& Chinese \% & 26.9 \\
& Indian \% & 9.4 \\
Age (yrs) & & $46.8 \pm 9.2$ \\
Weight $(\mathrm{kg})$ & & $63.7 \pm 10.5$ \\
Height (m) & & $1.60 \pm 0.078$ \\
BMI (kg/m²) & & $24.8 \pm 3.56$ \\
Waist (cm) & & $82.2 \pm 9.50$ \\
Systolic blood pressure $(\mathrm{mmHg})$ & & $126 \pm 6.6$ \\
Diastolic blood pressure $(\mathrm{mmHg})$ & & $78 \pm 9.0$ \\
Fasting C-peptide (pmol/l) & & $800 \pm 444$ \\
Fasting glucose (mmol/l) & & $4.9 \pm 0.56$ \\
Triglyceride (mmol/l) & & $1.08 \pm 0.54$ \\
Total cholesterol (mmol/l) & $4.7 \pm 0.88$ \\
HDL cholesterol (mmol/l) & $1.29 \pm 0.33$ \\
LDL cholesterol (mmol/l) & $2.89 \pm 0.84$ \\
Insulin resistance & $1.8 \pm 1.0$ \\
\hline
\end{tabular}


Table 2. Association of rs4810424 with metabolic syndrome parameters

\begin{tabular}{|c|c|c|c|c|c|c|}
\hline \multirow{2}{*}{$\begin{array}{l}\text { Metabolic syndrome } \\
\text { Parameter }\end{array}$} & \multicolumn{6}{|c|}{ Rs4810424 } \\
\hline & $\begin{array}{c}C C \\
\mathrm{n}(40)\end{array}$ & $\begin{array}{c}\mathrm{CG} \\
\mathrm{n}(79)\end{array}$ & $\begin{array}{c}\mathrm{GG} \\
\mathrm{n}(35)\end{array}$ & $\begin{array}{c}\text { Multivariate } \\
\text { P-value }\end{array}$ & $\begin{array}{c}\text { GG versus } C C \\
\mathrm{P} \text {-value }\end{array}$ & $\begin{array}{c}\text { GG versus CG } \\
\mathrm{P} \text {-value }\end{array}$ \\
\hline Insulin resistance & $1.87(1.61-2.17)$ & $1.56(1.39-2.74)$ & $1.41(1.17-1.68)$ & 0.039 & 0.017 & 0.36 \\
\hline C-peptide & 796 (674-939) & $679(604-764)$ & $621(520-742)$ & & 0.046 & 0.413 \\
\hline Fasting blood glucose & $4.95(4.8-5.1)$ & $4.92(4.8-5.0)$ & $4.74(4.59-4.90)$ & & 0.055 & 0.060 \\
\hline Triacylglycerol & $1.07(0.95-1.21)$ & $1.0(0.91-1.09)$ & $0.99(0.86-1.12)$ & & 0.34 & 0.89 \\
\hline HDL cholesterol & $1.22(1.15-1.31)$ & $1.26(1.20-1.30)$ & $1.34(1.25-1.44)$ & & 0.060 & 0.14 \\
\hline Waist & $81.1(78.5-83.7)$ & $82.3(80.4-84.2)$ & 79.89 (77.2-82.7) & & 0.530 & 0.158 \\
\hline Body mass index & $24.8(23.7-25.9)$ & $24.8(24.0-25.5)$ & $23.3(22.2-24.4)$ & & 0.066 & 0.036 \\
\hline Total Cholesterol & $4.7(4.5-5.0)$ & $4.6(4.3-4.7)$ & $4.5(4.3-4.7)$ & 0.317 & 0.22 & 0.48 \\
\hline LDL cholesterol & $2.9(2.7-3.2)$ & $2.8(2.6-2.9)$ & $2.6(2.3-2.8)$ & & 0.056 & 0.18 \\
\hline Systolic blood pressure & $123(119-127)$ & $125(122-127)$ & 123 (119-127) & 0.672 & 0.91 & 0.47 \\
\hline Diastolic blood pressure & $78(75-80)$ & $78(76-80)$ & $76(74-79)$ & & 0.37 & 0.22 \\
\hline
\end{tabular}

The results are presented as geometric mean and $95 \%$ confidence interval of the mean adjusted for age, sex and race as covariate.

homozygous GG variant had significantly lower insulin resistance $(\mathrm{P}=0.017)$ and $\mathrm{C}$-peptide levels $(\mathrm{P}=0.046)$ than those with homozygous CC. In addition, the FBG, BMI and LDLc were non-significantly lower in subjects having the homozygous minor GG variant than the homozygous major CC variant $(\mathrm{P}=0.055,0.06,0.056$, respectively), whereas HDLc was non-significantly higher $(\mathrm{P}=0.06)$. The subjects having heterozygous $\mathrm{CG}$ of this SNP showed a significantly higher BMI $(\mathrm{P}=0.036)$ and non-significantly higher FBG $(\mathrm{P}=0.06)$ than the subjects carrying homozygous minor GG. The multivariate analysis showed that homozygous minor and heterozygous variants of this SNP were not associated with waist, TG, total cholesterol, systolic and diastolic blood pressure.

\section{Association of P2 promoter SNP rs1884613 with metabolic syndrome parameters}

Multivariate analyses of this SNP with the three sets of metabolic syndrome parameters showed a non-significant Wilks' Lambda test with the first set (insulin resistance, C-peptide, FBG, TG, HDLc, waist and BMI), the second set (total and LDL cholesterol) and the third set $(\mathrm{P}=0.131,0.347,0.282$, respectively) (Table 3$)$. Parameter estimates of the multivariate test showed that subjects carrying homozygous minor variant for this SNP had lower insulin resistant and FBG, and higher HDLc than subjects carrying homozygous major variant $(\mathrm{P}=0.037,0.042,0.038$, respectively). The subjects carrying the heterozygous (CG) variant for this SNP had lower HDLc $(\mathrm{P}=0.042)$ and higher BMI (0.035) than those with homozygous minor variant. The homozygous major and heterozygous variants of this SNP were not associated with the other metabolic syndrome parameters (Cpeptide, TG, waist, total and LDL cholesterol, systolic and diastolic blood pressure).

\section{Association of P2 promoter SNP rs1884614 with metabolic syndrome parameters}

The SNP multivariate analyses of the three sets of metabolic syndrome parameters showed a non-significant Wilks' Lambda test with the first set (insulin resistance, C-peptide, FBG, TG, HDLc, waist and BMI), the second set (total and LDL cholesterol) and the third set (systolic and diastolic blood pressure $)(P=0.112,0.243,0.395$,

Table 3. Association of rs 1884613 with metabolic syndrome parameters

\begin{tabular}{|c|c|c|c|c|c|c|}
\hline \multirow{2}{*}{$\begin{array}{l}\text { Metabolic syndrome } \\
\text { parameter }\end{array}$} & \multicolumn{6}{|c|}{ Rs1884613 } \\
\hline & $\begin{array}{c}\mathrm{CC} \\
\mathrm{n}(52)\end{array}$ & $\begin{array}{c}\text { CG } \\
\mathrm{n}(68)\end{array}$ & $\begin{array}{c}\mathrm{GG} \\
\mathrm{n}(34)\end{array}$ & $\begin{array}{l}\text { Multivariate } \\
\text { P-value }\end{array}$ & $\begin{array}{c}\text { GG versus CC } \\
\text { P-value }\end{array}$ & $\begin{array}{c}\text { GG versus CG } \\
\text { P-value }\end{array}$ \\
\hline Insulin resistance & $1.79(1.56-2.04)$ & $1.56(1.38-1.76)$ & 1.17 (1.17-1.68) & 0.131 & 0.037 & 0.35 \\
\hline C-peptide & 763 (659-882) & $685(603-778)$ & $614(513-736)$ & & 0.068 & 0.33 \\
\hline Fasting blood glucose & $5.0(4.9-5.1)$ & $4.9(4.8-5.0)$ & $4.8(4.6-4.9)$ & & 0.042 & 0.29 \\
\hline Triacylglycerol & $1.01(0.90-1.13)$ & $1.03(0.93-1.13)$ & $0.98(0.85-1.11)$ & & 0.68 & 0.55 \\
\hline HDL cholesterol & $1.24(1.16-1.31)$ & 1.24 1.20-1.32) & $1.34(1.25-1.44)$ & & 0.038 & 0.042 \\
\hline Waist & $81.1(78.5-83.7)$ & $82.3(80.4-84.2)$ & $79.9(77.2-82.7)$ & & 0.40 & 0.18 \\
\hline Body mass index & $24.8(23.7-25.9)$ & $24.8(24.0-25.5)$ & $23.3(22.2-24.4)$ & & 0.12 & 0.035 \\
\hline Total cholesterol & $4.7(4.4-4.9)$ & $4.5(4.4-4.7)$ & $4.5(4.3-4.8)$ & 0.347 & 0.49 & 0.96 \\
\hline LDL cholesterol & $2.9(2.7-3.1)$ & $2.7(2.5-2.9)$ & $2.6(2.4-2.9)$ & & 0.12 & 0.60 \\
\hline Systolic blood pressure & $123(119-127)$ & $125(122-128)$ & $123(119-128)$ & 0.282 & 0.86 & 0.56 \\
\hline Diastolic blood pressure & $77(75-79)$ & $78(77-80)$ & 76 73-78) & & 0.34 & 0.076 \\
\hline
\end{tabular}

The results are presented as geometric mean and $95 \%$ confidence interval of the mean adjusted for age, sex and race as covariate. 
Table 4. Association of rs1884614 with metabolic syndrome parameters

\begin{tabular}{|c|c|c|c|c|c|c|}
\hline \multirow{2}{*}{$\begin{array}{l}\text { Metabolic syndrome } \\
\text { parameter }\end{array}$} & \multicolumn{6}{|c|}{ rs1884614 } \\
\hline & $\begin{array}{l}\mathrm{CC} \\
\mathrm{n}(46)\end{array}$ & $\begin{array}{c}\mathrm{CT} \\
\mathrm{n}(72)\end{array}$ & $\underset{n(33)}{T}$ & $\begin{array}{l}\text { Multivariate } \\
\text { P-value }\end{array}$ & $\begin{array}{l}\text { TT versus CC } \\
\text { P-value }\end{array}$ & $\begin{array}{l}\text { TT versus } \mathrm{CT} \\
\mathrm{P} \text {-value }\end{array}$ \\
\hline Insulin resistance & $1.78(1.53-2.05)$ & $1.63(1.44-1.84)$ & $1.35(1.10-1.62)$ & 0.112 & 0.024 & 0.087 \\
\hline C-peptide & $761(650-892)$ & $716(632-812)$ & 577 (479-695) & & 0.028 & 0.059 \\
\hline Fasting blood glucose & $4.9(4.8-5.1)$ & $4.9(4.8-5.0)$ & $4.8(4.6-4.9)$ & & 0.16 & 0.43 \\
\hline Triacylglycerol & $1.05(0.93-1.17)$ & $1.01(0.92-1.12)$ & $1.00(0.87-1.15)$ & & 0.66 & 0.93 \\
\hline HDL cholesterol & $1.24(1.17-1.32)$ & $1.26(1.19-1.32)$ & $1.36(1.27-1.46)$ & & 0.057 & 0.060 \\
\hline Waist & $81.9(79.5-84.4)$ & $81.5(79.6-83.5)$ & $80.4(77.6-83.3)$ & & 0.43 & 0.53 \\
\hline Body mass index & $24.9(23.9-25.9)$ & $24.6(23.8-25.4)$ & $23.3(22.2-24.4)$ & & 0.039 & 0.061 \\
\hline Total cholesterol & $4.7(4.5-4.9)$ & $4.6(4.4-4.8)$ & $4.5(4.3-4.8)$ & 0.243 & 0.39 & 0.75 \\
\hline LDL cholesterol & $2.9(2.7-3.2)$ & $2.7(2.6-2.9)$ & $2.6(2.3-2.8)$ & & 0.073 & 0.320 \\
\hline Systolic blood pressure & $123(120-127)$ & $126(123-129)$ & $121(116-125)$ & 0.395 & 0.36 & 0.059 \\
\hline Diastolic blood pressure & 77 (75-79) & $78(76-70)$ & $76(73-78)$ & & 0.32 & 0.16 \\
\hline
\end{tabular}

The results are presented as geometric mean and 95\% confidence interval of the mean adjusted for age, sex and race as covariate.

respectively) (Table 4). However, parameter estimates of multivariate analyses showed that insulin resistance, Cpeptide and BMI were significant lower in subjects having homozygous minor TT variant than the homozygous major CC subjects $(\mathrm{P}=0.024,0.028,0.039$, respectively). The subjects having homozygous major or heterozygous variant had non-significantly lower HDLc than those with the homozygous minor variant $(\mathrm{P}=0.057,0.060$, respectively). The heterozygous and homozygous major variants were not associated with the other metabolic syndrome parameters but the pattern was similar to that for HDLc.

\section{Association of intron 1D SNP rs2144908 with metabolic syndrome parameters}

The dependent variables of the first set (insulin resistance, C-peptide, FBG, TG, HDLc, waist and BMI) showed a significant Wilks' Lambda test $(\mathrm{P}=0.036)$ although the parameter estimates of the multivariate analysis showed that only HDLc was significantly higher among subjects having the homozygous minor (AA) variant than in those carrying the homozygous major one $(\mathrm{P}=0.020)$ (Table 5). In addition, HDLc was border line higher in the subjects having one copy of this SNP minor variant than those subjects having no minor genotype copies $(\mathrm{P}=0.054)$. Wilks' Lambda test for the variables of the second set (total and LDL cholesterol) and the third set (systolic and diastolic blood pressure) were non-significant $(P=0.73,0.258$, respectively) as well as the parameter estimates showed no association of these variables with rs2144908 genotypes.

\section{Association of intron SNP rs3818247 with metabolic syndrome parameters}

The multivariate analyses of the three sets of metabolic syndrome parameters indicated a non-significant Wilks' Lambda test with the first set (insulin resistance, C-peptide, FBG, TG, HDLc, waist and BMI), the second set (total and LDL cholesterol) and the third set (systolic and diastolic blood pressure) (Table 6). The parameter estimates indicated that systolic and diastolic blood pressure were higher among subjects carrying the

Table 5. Association of rs2144908 with metabolic syndrome parameters

\begin{tabular}{|c|c|c|c|c|c|c|}
\hline \multirow{2}{*}{$\begin{array}{l}\text { Metabolic syndrome } \\
\text { parameter }\end{array}$} & \multicolumn{6}{|c|}{ rs2144908 } \\
\hline & $\begin{array}{c}\mathrm{GG} \\
\mathrm{n}(54)\end{array}$ & $\begin{array}{l}\mathrm{AG} \\
\mathrm{n}(70)\end{array}$ & $\begin{array}{c}\mathrm{AA} \\
\mathrm{n}(33)\end{array}$ & $\begin{array}{l}\text { Multivariate } \\
\text { P-value }\end{array}$ & $\begin{array}{c}\text { AA versus GG } \\
\text { P-value }\end{array}$ & $\begin{array}{c}\text { AA versus AG } \\
\text { P-value }\end{array}$ \\
\hline Insulin resistance & $1.70(1.48-1.94)$ & $1.59(1.40-1.79)$ & $1.52(1.26-1.81)$ & 0.036 & 0.33 & 0.69 \\
\hline C-peptide & $728(630-840)$ & $690(608-783)$ & 664 (551-799) & & 0.44 & 0.73 \\
\hline Fasting blood glucose & $4.9(4.8-5.1)$ & $4.9(4.8-5.0)$ & $4.8(4.6-4.9)$ & & 0.090 & 0.21 \\
\hline Triacylglycerol & $0.97(0.85-1.06)$ & $1.04(0.94-1.14)$ & $1.04(0.91-1.19)$ & & 0.32 & 0.94 \\
\hline HDL cholesterol & $1.23(1.16-1.30)$ & $1.25(1.19-1.32)$ & $1.37(1.27-1.46)$ & & 0.020 & 0.054 \\
\hline Waist & $80.7(78.5-82.9)$ & $82.0(80.1-84.1)$ & $81.7(78.8-84.7)$ & & 0.58 & 0.87 \\
\hline Body mass index & $24.4(23.5-25.4)$ & $24.7(23.9-25.5)$ & $23.8(22.6-25.0)$ & & 0.39 & 0.22 \\
\hline Total cholesterol & $4.5(4.3-4.7)$ & $4.6(4.4-4.8)$ & $4.6(4.4-4.9)$ & 0.731 & 0.51 & 0.78 \\
\hline LDL cholesterol & $2.8(2.6-3.0)$ & $2.8(2.6-2.9)$ & $2.6(2.4-2.9)$ & & 0.38 & 0.50 \\
\hline Systolic blood pressure & $122(118-125)$ & $126(123-129)$ & 123 (119-127) & 0.258 & 0.66 & 0.24 \\
\hline Diastolic blood pressure & $76(75-78)$ & $79(77-80)$ & $76(74-78)$ & & 0.75 & 0.089 \\
\hline
\end{tabular}

The results are presented as geometric mean and 95\% confidence interval of the mean adjusted for age, sex and race as covariate. 
Table 6. Association of rs3818247 with metabolic syndrome parameters

\begin{tabular}{|c|c|c|c|c|c|c|}
\hline \multirow{2}{*}{$\begin{array}{l}\text { Metabolic syndrome } \\
\text { parameter }\end{array}$} & \multicolumn{6}{|c|}{ Rs3818247 } \\
\hline & $\prod_{n(54)}$ & $\begin{array}{c}\mathrm{GT} \\
\mathrm{n}(80)\end{array}$ & $\begin{array}{c}\mathrm{GG} \\
\mathrm{n}(23)\end{array}$ & $\begin{array}{l}\text { Multivariate } \\
\text { P-value }\end{array}$ & $\begin{array}{l}\text { GG versus } T T \\
\text { P-value }\end{array}$ & $\begin{array}{c}\text { GG versus GT } \\
\text { P-value }\end{array}$ \\
\hline Insulin resistance & $1.50(1.29-1.73)$ & $1.65(1.47-1.85)$ & $1.79(1.44-2.18)$ & 0.931 & 0.18 & 0.51 \\
\hline C-peptide & $644(557-746)$ & $722(641-813)$ & 766 (612-957) & & 0.21 & 0.65 \\
\hline Fasting blood glucose & $4.9(4.8-5.1)$ & $4.9(4.8-5.0)$ & $4.9(4.7-5.1)$ & & 0.42 & 0.91 \\
\hline Triacylglycerol & $1.04(0.93-1.15)$ & $1.00(0.91-1.09)$ & $1.01(0.85-1.18)$ & & 0.81 & 0.92 \\
\hline HDL cholesterol & $1.28(1.20-1.35)$ & $1.27(1.21-1.33)$ & $1.24(1.13-1.35)$ & & 0.58 & 0.62 \\
\hline Waist & $80.5(78.3-82.8)$ & $81.7(79.8-83.5)$ & $83.3(79.8-86.9)$ & & 0.19 & 0.42 \\
\hline Body mass index & $24.0(23.1-25.0)$ & $24.6(23.8-25.4)$ & $24.8(23.4-26.3)$ & & 0.39 & 0.78 \\
\hline Total cholesterol & $4.5(4.3-4.7)$ & $4.6(4.4-4.8)$ & $4.7(4.4-5.0)$ & 0.504 & 0.45 & 0.80 \\
\hline LDL cholesterol & $2.6(2.4-2.8)$ & $2.8(2.6-3.0)$ & $2.9(2.6-3.3)$ & & 0.15 & 0.50 \\
\hline Systolic blood pressure & $122(118-125)$ & $124(121-127)$ & $129(124-135)$ & 0.200 & 0.020 & 0.078 \\
\hline Diastolic blood pressure & $76(74-78)$ & $78(76-79)$ & $80(77-83)$ & & 0.034 & 0.17 \\
\hline
\end{tabular}

The results are presented as geometric mean and $95 \%$ confidence interval of the mean adjusted for age, sex and race as covariate.

Table 7. Association of most common haplotypes 2 and haplotypes 1 with metabolic syndrome parameters

\begin{tabular}{|c|c|c|c|c|}
\hline \multirow[b]{2}{*}{$\begin{array}{l}\text { Metabolic syndrome para- } \\
\text { meter }\end{array}$} & \multicolumn{4}{|c|}{ Most common haplotypes 2} \\
\hline & $\begin{array}{l}\text { CCCGTC } \\
\mathrm{n}(72)\end{array}$ & $\begin{array}{l}\text { GGTATC } \\
\mathrm{n}(46)\end{array}$ & $\begin{array}{l}\text { Multivariate } \\
\text { P-value }\end{array}$ & $\begin{array}{c}\text { CCCGTC } \\
\text { versus } \\
\text { GGTATC } \\
\text { P-value }\end{array}$ \\
\hline Insulin resistance & $1.78(1.59-1.98)$ & $1.43(1.22-1.66)$ & 0.002 & 0.022 \\
\hline C-peptide & $770(682-870)$ & $630(541-734)$ & & 0.044 \\
\hline Fasting blood glucose & $4.9(4.8-5.0)$ & $4.7(4.6-4.9)$ & & 0.035 \\
\hline Triacylglycerol & $1.07(0.98-1.16)$ & $0.97(0.87-1.09)$ & & 0.19 \\
\hline HDL cholesterol & $1.20(1.14-1.27)$ & $1.37(1.29-1.46)$ & & 0.001 \\
\hline Waist & $82.4(80.4-84.5)$ & $81.4(78.9-84.0)$ & & 0.544 \\
\hline Body mass index & $25.0(24.2-25.9)$ & $23.8(22.9-24.9)$ & & 0.078 \\
\hline Total cholesterol & $4.6(4.4-4.8)$ & $4.6(4.4-4.8)$ & 0.114 & 0.92 \\
\hline LDL cholesterol & $2.8(2.6-3.0)$ & $2.7(2.5-2.9)$ & & 0.31 \\
\hline Systolic blood pressure & $124(121-127)$ & $123(120-127)$ & 0.730 & 0.86 \\
\hline \multirow[t]{2}{*}{ Diastolic blood pressure } & $78(76-79)$ & $77(75-79)$ & & 0.52 \\
\hline & \multicolumn{4}{|c|}{ Most common haplotypes 1} \\
\hline $\begin{array}{l}\text { Metabolic syndrome para- } \\
\text { meter }\end{array}$ & $\begin{array}{l}\text { CCCGTC } \\
\mathrm{n}(39)\end{array}$ & $\begin{array}{l}\text { GGTATC } \\
\mathrm{n}(68)\end{array}$ & $\begin{array}{l}\text { Multivariate } \\
\text { P-value }\end{array}$ & $\begin{array}{c}\text { CCCGTC } \\
\text { versus } \\
\text { GGTATC } \\
\text { P-value }\end{array}$ \\
\hline Insulin resistance & $1.71(1.46-1.97)$ & $1.56(1.38-1.75)$ & 0.161 & 0.34 \\
\hline C-peptide & $731(622-860)$ & $681(602-770)$ & & 0.70 \\
\hline Fasting blood glucose & $5.0(4.8-5.1)$ & $4.9(4.8-5.0)$ & & 0.37 \\
\hline Triacylglycerol & $0.95(0.83-1.08)$ & $1.05(0.95-1.15)$ & & 0.22 \\
\hline HDL cholesterol & $1.25(1.17-1.34)$ & $1.28(1.21-1.34)$ & & 0.65 \\
\hline Waist & $80.1(77.7-82.6)$ & $81.3(79.4-83.2)$ & & 0.56 \\
\hline Body mass index & $24.6(23.5-25.6)$ & $24.3(23.5-25.1)$ & & 0.72 \\
\hline Total cholesterol & $4.7(4.5-4.9)$ & $4.6(4.4-4.8)$ & 0.178 & 0.51 \\
\hline LDL cholesterol & $2.9(2.7-3.2)$ & $2.7(2.5-2.9)$ & & 0.15 \\
\hline Systolic blood pressure & $124(120-128)$ & $125(122-128)$ & 0.872 & 0.60 \\
\hline Diastolic blood pressure & 77 (75-79) & $78(76-79)$ & & 0.71 \\
\hline
\end{tabular}

The results are presented as geometric mean and $95 \%$ confidence interval of the mean adjusted for age, sex and race as covariate. 
Table 8. Association of most common diplotypes with metabolic syndrome parameters

\begin{tabular}{|c|c|c|c|c|c|c|}
\hline \multirow[b]{2}{*}{$\begin{array}{l}\text { Metabolic syndrome } \\
\text { parameter }\end{array}$} & \multicolumn{6}{|c|}{ Most common diplotypes } \\
\hline & $\begin{array}{c}\text { CCCGTC, } \\
\text { CCCGTC } \\
\text { (Diplotype No. 1) } \\
\mathrm{n}(21)\end{array}$ & $\begin{array}{c}\text { GGTATC, } \\
\text { CCCGTC } \\
\text { (Diplotype No. 2) } \\
\text { n( } 36)\end{array}$ & $\begin{array}{c}\text { GGTATC, } \\
\text { GGTATC } \\
\text { (Diplotype No. 3) } \\
\text { n(24) }\end{array}$ & $\begin{array}{l}\text { Multivariate } \\
\text { P-value }\end{array}$ & $\begin{array}{c}\text { Diplotype No1 } \\
\text { versus } \\
\text { Diplotype No } 3 \\
\text { P-value }\end{array}$ & $\begin{array}{c}\text { Diplotype No } 2 \\
\text { versus } \\
\text { Diplotype No } 3 \\
\text { P-value }\end{array}$ \\
\hline Insulin resistance & $1.89(1.54-2.27)$ & $1.74(1.49-2.02)$ & $1.38(1.12-1.68)$ & 0.064 & 0.030 & 0.071 \\
\hline C-peptide & $810(652-1007)$ & 766 (649-904) & $607(495-744)$ & & 0.058 & 0.083 \\
\hline Fasting blood glucose & $5.0(4.8-5.2)$ & $5.0(4.8-5.1)$ & $4.8(4.6-4.9)$ & & 0.11 & 0.11 \\
\hline Triacylglycerol & $1.05(0.88-1.23)$ & $1.10(0.97-1.24)$ & $1.03(0.88-1.20)$ & & 0.91 & 0.54 \\
\hline HDL cholesterol & $1.21(1.10-1.33)$ & $1.19(1.10-1.28)$ & $1.37(1.25-1.49)$ & & 0.065 & 0.018 \\
\hline Waist & $79.9(76.5-83.4)$ & $82.6(79.9-85.4)$ & $80.4(77.2-83.7)$ & & 0.83 & 0.31 \\
\hline Body mass index & $24.6(23.2-26.0)$ & $24.9(23.9-26.0)$ & $23.34(22.1-24.7)$ & & 0.20 & 0.070 \\
\hline Total cholesterol & $4.7(4.4-5.0)$ & $4.6(4.3-4.8)$ & $4.7(4.4-5.0)$ & 0.402 & 0.99 & 0.63 \\
\hline LDL cholesterol & $2.9(2.6-3.3)$ & $2.8(2.5-3.0)$ & $2.7(2.4-3.0)$ & & 0.33 & 0.65 \\
\hline Systolic blood pressure & $121(116-127)$ & $126(122-130)$ & $122(117-127)$ & 0.446 & 0.85 & 0.19 \\
\hline Diastolic blood pressure & $76(73-79)$ & $79(76-81)$ & $76(73-79)$ & & 0.84 & 0.16 \\
\hline
\end{tabular}

The results are presented as geometric mean and 95\% confidence interval of the mean adjusted for age, sex and race as covariate.

homozygous minor (GG) variant than the subjects carrying the homozygous major (T'T) $(0.02,0.034$, respectively).

\section{Associations of common haplotypes with metabolic syndrome parameters}

Six-SNP haplotype and diplotype blocks were identified with significant LD. This block is constructed from rs24 (rs4810424), rs13 (rs1884613), rs14 (rs1884614), rs08 (rs2144908), rs51 (rs6031551) and rs52 (rs6031552) (Fig. 1). Wilks' Lambda test of multivariate analysis showed that the most common haplotypes 2 were associated with the first set of metabolic syndrome parameters (insulin resistance, C-peptide, FBG, TG, HDLc, waist and $\mathrm{BMI})(\mathrm{P}=0.002)$. The parameter estimates of this multivariate analysis indicated that subjects carrying the haplotype2, GGTATC, had higher HDLc and lower insulin resistance, $\mathrm{C}$-peptide and $\mathrm{FBG}$ than subjects carrying the haplotpe2, CCCGTC $(\mathrm{P}=0.001,0.022,0.044$, 0.035) (Table 7). Wilks' Lambda tests and parameter estimates of multivariate analyses of the second set (total and LDL cholesterol) and the third set (systolic and diastolic blood pressure) of metabolic syndrome param-
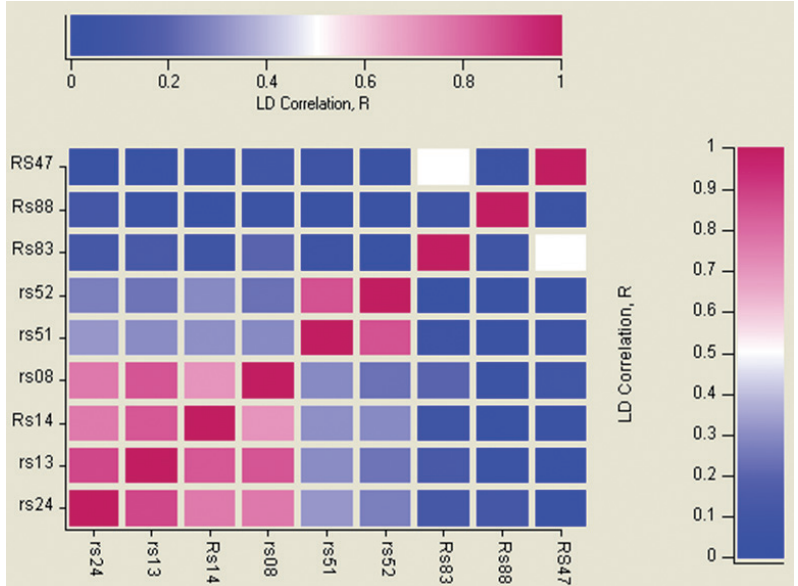

Figure 1. Linkage disequilibrium correlation between SNPs rs24, rs4810424; rs13, rs1884613; rs14, rs1884614; rs08, rs2144908; rs51, rs6031551; rs52, rs6031552. eters were not associated with haplotypes2. The most common haplotypes1 (CCCGTC and GGTATC) were not associated with the three sets of metabolic syndrome parameters, however, these haplotypes1 showed a similar pattern to the most common haplotypes 2 .

\section{Association of the common diplotypes with metabolic syndrome parameters}

The association of the most common diplotypes with the first set of metabolic syndrome parameters (insulin resistance, C-peptide, FBG, TG, HDLc, waist and BMI) was border line (Wilks' Lambda test p-value $=0.064$ ) (Table 8). The parameter estimates of these metabolic syndrome parameters indicated that subjects carrying the GGTATC,GGTATC diplotype were less insulin resistant than the subjects carrying the CCCGTC,CCCGTC or GGTATC,CCCGTC diplotype ( $\mathrm{P}=0.030,0.071$, respectively). In addition, HDLc was higher in subjects having the GGTATC,GGTATC diplotype than in subjects having the CCCGTC,GGTATC or CCCGTC,CCCGTC diplotype ( $\mathrm{P}=0.018,0.065$, respectively). Wilks' Lambda tests and the parameter estimates of total and LDL cholesterol, systolic and diastolic blood pressure showed that these parameters were not associated with the above most common diplotypes.

\section{DISCUSSION}

The results showed that the homozygous major variants for the HNF4 alpha P2 promoter SNPs (rs4810424, rs1884614 and rs1884613) associated with insulin resistance, C-peptide and BMI whereas the intron 1D SNP rs2144908 associated with HDL cholesterol. In addition, the haplotypes2 (CCCGTC) comprising the homozygous major variants of rs4810424, rs1884613, rs1884614, and rs2144908 showed an additional significant association with insulin resistance, C-peptide, fasting blood glucose and HDL cholesterol. The individuals carrying homozygous major variants for these SNPs might be at a higher risk of developing metabolic syndrome (prediabetic stage) and hence T2DM. The risk for developing T2DM will be increased in the subjects carrying the risk haplotype (CCCGTC). 
Andrulionyte et al. (2006) found that female carriers of the C allele of rs 4810424 had a 1.7 -fold elevated risk for the conversion to diabetes compared to woman with the common genotype. Recently, a large cohort study (17831 individuals from Sweden and Finland) has reported that homozygous CC of rs4810424 predicts further T2DM in individuals from Botnia Study in Sweden (Holmkvist et al., 2008). Hansen et al. (2005) found that homozygous minor (TT) individuals for rs1884614 had non-significantly lower insulin resistance, C-peptide and $\mathrm{BMI}$ than homozygous major CC individuals. In contrast, Holmkvist et al. (2008) reported that the rs1884614 homozygous minor TT genotype predicted further T2DM in the Botnia Study (Sweden) in T2DM relative subjects (Holmkvist et al., 2008). Wanic et al. (2006) and Holmkvist et al. (2008) found that the intron 1D SNP rs2144908 did not associate with diabetic parameters (insulin resistance, C-peptide and glucose). In contrast, Silander et al. (2004) reported that the homozygous AA of this SNP was associated with low BMI, acute insulin response to glucose and glucose disposition index, high fasting insulin, and high differences between 2-hour and fasting glucose and insulin during oral glucose tolerance test (OGTT).

The discrepancies in the association study of HNF4 alpha SNPs and metabolic parameters such as glucose, insulin, insulin resistance and BMI may be due to the characteristics of the subjects. The Hansen et al. (2005) subjects were normal glucose-tolerant whereas Silander et al. (2004) and Holmkvist et al. (2008) subjects were unaffected offspring of T2DM and family related T2DM, respectively. On the other hand, the mean BMI of normal subjects in the Wanic et al. (2006) study was high $\left(30 \mathrm{~kg} / \mathrm{m}^{2}\right)$, which markedly affected the metabolic syndrome parameters. Low frequencies of HNF4 alpha SNPs homozygous minor genotypes in some populations resulted in too few subjects within the subgroups, for example 14 subjects with homozygous AA in the Silander et al. (2004) study that resulted in low power of statistical analysis.

The HNF4 alpha P2 promoter risk variants showed higher fasting glucose, which could be due to increased hepatic cell glucose production. This suggestion was supported by the Holmkvist et al. (2008) finding that HNF4 P2 promoter variants were associated with increased hepatic glucose production. This increased blood glucose was compensated by increased pancreatic beta cell insulin secretion (C-peptide was high in the HNF4 alpha P2 risk variants). However, the hepatic response to that secreted insulin might be slightly impaired, resulting in insulin resistance in the individuals carrying the HNF4 P2 promoter risk variants. The beta cell responsiveness to the increased blood glucose would be reduced in the long term until insulin secretion decreased more than $50 \%$, resulting in the diagnosis of T2DM. This suggestion was confirmed by the Lehman et al. (2007) finding that the age of T2DM onset was decreased in HNF4 alpha P2 promoter risk variants.

The non-coding variants of $\mathrm{HNF} 4$ alpha may exert effects on gene expression (Bonnycastle et al., 2006). It was found that HNF4 alpha was associated with a large number of promoters in the liver, an important organ for glucose production, and pancreatic islet cells responsible for insulin secretion (Odom et al., 2004). HNF4 alpha plays an important role in regulating glucose metabolism, hepatic gluconeogenesis (Stoffel \& Duncan, 1997; Puigserver et al., 2003; Rhee et al., 2003), and lipoprotein and cholesterol metabolism (Rhee et al., 2006).
In conclusion, HNF4 alpha P2 promoter SNPs were associated with insulin resistance, C-peptide and BMI whereas the intron 1D SNP rs2144908 associated with HDL cholesterol. The haplotype comprising the P2 promoter and intronic 1D SNPs confirmed these associations. Individuals carrying the risk genotypes for the HNF4 alpha P2 promoter SNPs might be at a higher risk of developing T2DM and the risk may be further aggravated with the haplotype that contains these risk genotype SNPs.

\section{Acknowledgements}

This research was supported by a research grant from University Kebangsaan Malaysia Medical Molecular Biology Institute (UMBI). We thank Prof. Dr. Maznah Ismail and her lab staff, Laboratory of Molecular Biomedicine, Institute of Bioscience, University Putra Malaysia for giving us the opportunity to run our samples on their DHPLC.

\section{REFERENCES}

Amed S, Daneman D, Mahmud FH, Hamilton J (2010) Type 2 diabetes in children and adolescents. Expert Rev Cardiovasc Ther 8: 393406.

Andrulionyte L, Laukkanen O, Chiasson JL, Laakso M (2006) Single nucleotide polymorphisms of the HNF4alpha gene are associated with the conversion to type 2 diabetes mellitus: the STOP-NIDDM trial. J Mol Med 84: 701-708.

Bolotin E, Liao H, Ta TC, Yang C, Hwang-Verslues W, Evans JR, Jiang T, Sladek FM (2010) Integrated approach for the identification of human hepatocyte nuclear factor 4alpha target genes using protein binding microarrays. Hepatology 51: 642-653.

Bonnycastle LL, Willer CJ, Conneely KN, Jackson AU, Burrill CP, Watanabe RM, Chines PS, Narisu N, Scott LJ, Enloe ST, Swift AJ, Duren WL, Stringham HM, Erdos MR, Riebow NL, Buchanan TA, Valle T'T, Tuomilehto J, Bergman RN, Mohlke KL, Boehnke M, Collins FS (2006) Common variants in maturity-onset diabetes of the young genes contribute to risk of type 2 diabetes in Finns. Diabetes 55: 2534-2540.

Cali AM, Caprio S (2008) Prediabetes and type 2 diabetes in youth: an emerging epidemic disease? Curr Opin Endocrinol Diabetes Obes 15: $123-127$.

Gonzalez FJ (2008) Regulation of hepatocyte nuclear factor 4 alphamediated transcription. Drug Metab Pharmacokinet 23: 2-7.

Haffner SM, Mykkanen L, Festa A, Burke JP, Stern MP (2000) Insulin-resistant prediabetic subjects have more atherogenic risk factors than insulin-sensitive prediabetic subjects: implications for preventing coronary heart disease during the prediabetic state. Circulation 101: 975-980.

Hansen SK, Rose CS, Glumer C, Drivsholm T, Borch-Johnsen K, Jorgensen T, Pedersen O, Hansen T (2005) Variation near the hepatocyte nuclear factor (HNF)-4alpha gene associates with type 2 diabetes in the Danish population. Diabetologia 48: 452-458.

Holmkvist J, Almgren P, Lyssenko V, Lindgren CM, Eriksson KF, Isomaa B, Tuomi T, Nilsson P, Groop L (2008) Common variants in maturity-onset diabetes of the young genes and future risk of type 2 diabetes. Diabetes 57: 1738-1744.

Holst-Schumacher I, Nunez-Rivas H, Monge-Rojas R, Barrantes-Santamaria M (2009) Components of the metabolic syndrome among a sample of overweight and obese Costa Rican school children. Food Nutr Bull 30: 161-170.

Leclercq IA, Da Silva Morais A, Schroyen B, Van Hul N, Geerts A (2007) Insulin resistance in hepatocytes and sinusoidal liver cells: mechanisms and consequences. J Hepatol 47: 142-156.

Lehman DM, Richardson DK, Jenkinson CP, Hunt KJ, Dyer TD, Leach RJ, Arya R, Abboud HE, Blangero J, Duggirala R, Stern MP (2007) P2 promoter variants of the hepatocyte nuclear factor 4alpha gene are associated with type 2 diabetes in Mexican Americans. Diabetes 56: 513-517.

Love-Gregory LD, Wasson J, Ma J, Jin CH, Glaser B, Suarez BK, Permutt MA (2004) A common polymorphism in the upstream promoter region of the hepatocyte nuclear factor- 4 alpha gene on chromosome $20 \mathrm{q}$ is associated with type 2 diabetes and appears to contribute to the evidence for linkage in an Ashkenazi jewish population. Diabetes 53: 1134-1140.

Nathan BM (2007) The increase of type 2 diabetes mellitus in children. Minn Med 90: 39-43. 
Odom DT, Zizlsperger N, Gordon DB, Bell GW, Rinaldi NJ, Murray HL, Volkert TL, Schreiber J, Rolfe PA, Gifford DK, Fraenkel E, Bell GI, Young RA (2004) Control of pancreas and liver gene expression by HNF transcription factors. Science 303: 1378-1381.

Parviz F, Matullo C, Garrison WD, Savatski L, Adamson JW, Ning G, Kaestner KH, Rossi JM, Zaret KS, Duncan SA (2003) Hepatocyte nuclear factor 4 alpha controls the development of a hepatic epithelium and liver morphogenesis. Nat Genet 34: 292-296.

Phillips J, Phillips PJ (2009) Children get type 2 diabetes too. Aust Fam Physician 38: 699-703.

Puigserver P, Rhee J, Donovan J, Walkey CJ, Yoon JC, Oriente F, Kitamura Y, Altomonte J, Dong $\mathrm{H}$, Accili D, Spiegelman BM (2003) Insulin-regulated hepatic gluconeogenesis through FOXO1PGC-1alpha interaction. Nature 423: 550-555.

Rhee J, Inoue Y, Yoon JC, Puigserver P, Fan M, Gonzalez FJ, Spiegelman BM (2003) Regulation of hepatic fasting response by PPARgamma coactivator-1alpha (PGC-1): requirement for hepatocyte nuclear factor 4alpha in gluconeogenesis. Proc Natl Acad Sci USA 100: 4012-4017.

Rhee J, Ge H, Yang W, Fan M, Handschin C, Cooper M, Lin J, Li C, Spiegelman BM (2006) Partnership of PGC-1alpha and HNF4alpha in the regulation of lipoprotein metabolism. I Biol Chem 281: 14683-14690.
Sesti G (2006) Pathophysiology of insulin resistance. Best Pract Res Clin Endocrinol Metab 20: 665-679.

Silander K, Mohlke KL, Scott LJ, Peck EC, Hollstein P, Skol AD, Jackson AU, Deloukas P, Hunt S, Stavrides G, Chines PS, Erdos MR, Narisu N, Conneely KN, Li C, Fingerlin TE, Dhanjal SK, Valle TT, Bergman RN, Tuomilehto J, Watanabe RM, Boehnke M, Collins FS (2004) Genetic variation near the hepatocyte nuclear factor-4 alpha gene predicts susceptibility to type 2 diabetes. Diabetes 53: 1141-1149.

Stoffel M, Duncan SA (1997) The maturity-onset diabetes of the young (MODY1) transcription factor HNF4alpha regulates expression of genes required for glucose transport and metabolism. Proc Natl Acad Sci USA 94: 13209-13214.

Wang H, Maechler P, Antinozzi PA, Hagenfeldt KA, Wollheim CB (2000) Hepatocyte nuclear factor 4alpha regulates the expression of pancreatic beta-cell genes implicated in glucose metabolism and nutrient-induced insulin secretion. J Biol Chem 275: 35953-35959.

Wanic K, Malecki MT, Wolkow PP, Klupa T, Skupien J, Bobrek J, Kozek E, Krolewski AS, Sieradzki J (2006) Polymorphisms in the gene encoding hepatocyte nuclear factor-4alpha and susceptibility to type 2 diabetes in a Polish population. Diabetes Metab 32: 86-88. 